\title{
Research of the Influence of Production Technologies on the Positioning Accuracy of a Robotic Arm for Low Handling Weights
}

\section{Peter Michalik}

Technical University of Košice with a seat in Prešov

Vieroslav Molnár ( $\square$ vieroslav.molnar@tuke.sk)

Technical University of Košice with a seat in Prešov

\section{Gabriel Fedorko}

Technical University of Košice

\section{Beáta Stehlíková}

Technical University of Košice

\section{Peter Tirpak}

Technical University of Košice with a seat in Prešov

\section{Jozef Macej}

Technical University of Košice with a seat in Prešov

\section{Research Article}

Keywords: robot, methodology, arm, position accuracy, verification

Posted Date: May 26th, 2021

DOI: https://doi.org/10.21203/rs.3.rs-494018/v1

License: (c) (1) This work is licensed under a Creative Commons Attribution 4.0 International License. Read Full License 


\section{Abstract}

The subject of the paper is the research of production technologies influence on positioning accuracy of a robotic arm. The aim was to find out whether different production technologies (additive and conventional) and the related design differences of the robotic arm affect its operational functionality. In the research, positioning accuracy of a robotic arm formed by 3 partial arms was specifically investigated, while the first partial arm Arm I was manufactured by two different technologies. On the robotic arm, the research was carried out in such a way that the first partial arm, Arm I, was being continuously changed and was available for research purposes in two variants. Each of the Arm I variants was manufactured using a different technology (additive and conventional) while, at the same time, the individual variants also differed in construction. The design differences of both variants were related to the production technology used. The measurement of positioning accuracy was performed with the use of two methods. Specifically, a contact and a non-contact method was used. The contact method was implemented on a 3D-measuring machine RAPID and the second contactless method was performed using an inductive sensor. The maximum working load of the robotic arm was $2 \mathrm{~kg}$, so the positioning accuracy was examined at three degrees of operating load equal to $0 ; 50$ and $100 \%$ of the maximum workload.

\section{Introduction}

The use of robots for handling and positioning, welding, subtractive and additive production has been expanding in recent years in line with the Industry 4.0 concept [1]. A robotic arm can be a separate mechanism or part of a more complex robot. It is a type of mechanical hand that is usually programmable and has similar functions to a human hand [2].

At present, we encounter the use of various production technologies in the production of robotic arm components. CNC machining technologies are the most widespread [3]. Covaciu and Filip [4] designed and validated a robotic arm made with two CNC machines. Gordaninejad and Vaidyaraman are among the first researchers to compare the positioning accuracy of robotic arms made of conventional metallic materials and advanced composites [5].

Until recently, it was not possible to find a published use of an additive manufacturing component in an industrial robotic arm. However, the great expansion of 3D printing technology is creating increasingly suitable conditions for this [6]. A conventional 3D printer uses layering in a horizontal plane to produce a 3D printed part. Ishak and Larochelle [7] proposed the integration of existing additive manufacturing process technologies with the arm of an industrial robot to create a multilayer layered 3D printer. Their approach allows printing to be layered in multiple planes, while existing conventional 3D printers are limited to one plane of the toolpath. The integration of the robotic arm and the extruder allows the movements of several planes of the tool path to be used in the production of structural parts. 
Hajash et al. [8] introduced a fast liquid printing device that can freely print in any direction, rather than layer by layer, depositing liquid material in a granular gel to create 3D structures. Recently, however, several scientific papers have appeared on the additive manufacturing of robotic arm components. Mick et al. [9] proposed a prototype of a robotic arm made using 3D printing, which is also economically more advantageous compared to the price of an industrial robot thanks to conventional drives. Junia Santillo Costa et al. [10] implemented and validated a 3D printed Open Source robotic arm with 6 degrees of freedom made of ABS material, due to higher mechanical strength. Ismail et al. [11] designed and developed a robotic arm for lifting light parts with 4 degrees of freedom. They used a 3D printing method to make the robotic arm components, which provided more accurate dimensions and time and cost savings. Wang et al. [12] proposed a special holder for the InnoMotion robotic arm, where the main components of the holder were made by 3D printing using plastic material and are fully compatible with the MR and CT system and the robotic arm.

The positioning accuracy of the robotic arm can vary widely in the workspace, which is influenced by various factors. Researchers therefore propose various methods and methodologies to improve reliability and repeatability of positioning accuracy [13]. One of the parameters influencing the accuracy of the robotic arm is vibration. Elvira-Ortiz et al. [14] proposed a methodology to improve the estimation of kinematic parameters on industrial robots by correctly suppressing the vibrational components present on the signals obtained from the two primary sensors: the accelerometer and the gyroscope. Their results prove that the sensor fusion technique, accompanied by correct vibration suppression, provides a better estimate of the kinematic parameters than other proposed techniques. The accuracy of the robotic arm is also affected by sensing the position of each joint with a high-resolution optical coding device that cannot detect certain mechanical deformations, thus reducing the accuracy of the robot's positioning and orientation. Research in this area has been addressed by Rodriguez-Donate et al. [15], who developed an intelligent processor using Kalman filters to filter and fuse information from a network of sensors. They used two primary sensors: an optical encoder and a 3-axis accelerometer. Calibration of robot arm is an important factor in the accuracy of robot positioning. A simple, low-cost calibration procedure that improves the surface positioning accuracy of a SCARA double-arm robot was published by Joubair et al. [16]. One of the key problems in examining the positioning accuracy of robotic arms is the working temperature. Kluz et al. Analyzed the analysis of the influence of temperature on the positioning accuracy of the robot arm [17]. The obtained results were subjected to statistical analysis using the Shapiro-Wilk test, which confirmed that the three-sigma rule can be used to calculate the value of the total positioning error of the robot arm.

The subject of the paper is the research of production technologies impact on positioning accuracy of a robotic arm. The aim was to find out whether different production technologies (additive and conventional) and the related design differences of the robotic arm affect its operational functionality. In the research, positioning accuracy of a robotic arm formed by 3 partial arms was specifically investigated, while the first partial arm Arm I was manufactured by two different technologies. On the robotic arm, the research was carried out in such a way that the first partial arm, Arm I, was being continuously changed and was available for research purposes in two variants. Each of the Arm I 
variants was manufactured using a different technology (additive and conventional) while, at the same time, the individual variants also differed in construction. The design differences of both variants were related to the production technology used. The measurement of positioning accuracy was performed with the use of two methods. Specifically, a contact and a non-contact method was used. The contact method was implemented on a 3D-measuring machine RAPID and the second contactless method was performed using an inductive sensor. The maximum working load of the robotic arm was $2 \mathrm{~kg}$, therefore the positioning accuracy was examined at three degrees of operating load equal to $0 ; 50$ and $100 \%$ of the maximum workload.

\section{Material And Methods}

The developed robot with the most used angular structure with rotational movements in 3 axes, Fig. 1, was used for the experiment.

The robot is in shape of a human arm with swivel joints. The working space consists of spherical areas. Such robots are suitable for a wide range of activities involving the use of three rotary motion axes. The robot itself consists of a robot base and three arms. The base of the robot is usually anchored horizontally, Arm I is mounted on it and rotates around the vertical axis Z. The remaining two axes of rotation are horizontal and parallel to each other. They consist of Arm II and Arm III. Arm III can work in proximity to the $Z$ axis. The individual arms of the robot are connected to each other by gear mechanisms driven by servo drives. As the arms move, their elastic deformation occurs, which also affects positioning accuracy of the end robotic arm with the manipulation effector. Positioning accuracy research was carried out on two identical versions of Arm I, which differed in production technology used.

\subsection{Arm I - made by additive manufacturing (AdM)}

Production of Arm I was realized by 3D printer Xline 2000R [18], used material is AlSi10Mg (Fig. 2).

Technical specification:

- layer height $0.06 \mathrm{~mm}$,

- support structure layer height $0.12 \mathrm{~mm}$,

- total time of finishing and removing of support structure, finishing and sandblasting 32 hours,

- printing time equals 39 hours and 35 minutes.

\subsection{Arm I - made by CNC milling (CvM)}

Production of Arm I was realized by CNC machine Pinnacle VMC 650S, used material is AIMg4.5Mn, DIN 1732, with dimensions $150 \mathrm{~mm} \times 150 \mathrm{~mm}-450 \mathrm{~mm}$ (Fig. 3). Its chemical composition is in Table 1 and physical-mechanical properties in Table 2. 
Table 1

Chemical composition according to EN573-3

\begin{tabular}{|llllllllll|}
\hline Si [\%] & Fe [\%] & Cu [\%] & Mn [\%] & Mg [\%] & Cr [\%] & Zn [\%] & \multicolumn{2}{c|}{ Ti [\%] } & \multicolumn{2}{c|}{ Others [\%] } \\
\hline max. & max. & max. & & & & $\max$. & $\max$. & $\max$. & $\max$. \\
\hline 0.40 & 0.40 & 0.10 & $0.40-1.0$ & $0.40-4.9$ & $0.05-0.25$ & 0.25 & 0.15 & 0.05 & 0.15 \\
\hline
\end{tabular}

Table 2

Physical-mechanical properties according to EN573-3

\begin{tabular}{|lllllll|}
\hline Fm [N] & Rp0.2 [MPa] & Rm [MPa] & Fmax [N] & a [mm] & b [mm] & E [GPa] \\
\hline 8841 & 198 & 242 & 8840 & 2.960 & 12.35 & 3.738 \\
\hline
\end{tabular}

Fig. 3. Arm I - CvM made on CNC machine Pinnacle VMC 650S.

\subsection{Contact measuring Method (CoM) of robotic arm's position}

CoM on a 3D-measuring machine can be implemented by a stylus with a ruby ball manually or by programming the stylus for repeated measurements. In our case, stylus programming by the learning method was used. Prior to measurement itself, the coordinate system of the 3D-measuring machine and the coordinate system of the measured - scanned body were identified. An NC scanning program was created in the control system of the 3D-measuring machine RAPID THOME Präzision [19], which ensured scanning of positions during repeated measurements. Stylus with a $4 \mathrm{~mm}$ diameter ruby ball was used for scanning as in Fig. 4. The defined scanning sensitivity of the 3D-measuring machine $0.001 \mathrm{~mm}$ was achieved by observing the design and operating conditions. A holder was mounted on the robotic arm, which allowed mounting and weight exchange, and at the same time a part of it was machined so that reading of three perpendicular planes is posible, which after alignment with the reference coordinate system 3D-measuring machine were used to sense the position.

The measuring chain for CoM is shown in Fig. 5. After setting the reference coordinate system of 3Dmeasuring machine (it consists in setting a fixed zero point on the base frame from which all measured values are read), the program for positioning the robotic arm is initialized and then adjusted to the position with maximum reach. The 3D-measuring machine is then initialized. The stylus with ruby ball automatically senses the end position of the robotic arm [mm] and loads it into the PC application Metrolog XG. In this application, the end position of the robotic arm is evaluated.

\subsection{Non-contact measuring Method (NcM) of robotic arm's position deviation}

In the second method of measuring position deviation, NcM was used using the proximity sensor MTN/EP080 Probe [20] in Fig. 6. The defined sensing sensitivity of the $0.01 \mathrm{~mm}$ proximity sensor was 
achieved by adhering to the design and operating conditions.

A holder with a replaceable weight machined in three planes perpendicular to each other was reused on the robotic arm. A stable stand made of non-magnetic material with a non-conductive proximity sensor holder was mounted on the base frame of a 3D-measuring machine. The holder with a replaceable weight is used (after setting the switching position of the proximity sensor) to sense the incremental position deviation in the direction of the $\mathrm{X}, \mathrm{Y}$ and $\mathrm{Z}$ axes in micrometers.

In the measuring chain (Fig. 7), the power supply to the proximity sensor is initialized first. Subsequently, the program for positioning the robotic arm is activated. This is followed by positioning the robotic arm to the position with maximum reach. After reaching this position, a graphical recording of the maximum reach of robotic arm measurement will be performed. The incremental value of position deviation for the maximum reach of the robotic arm in $\mu \mathrm{m}$ is read from the graphic record in the LabVIEW software.

Robotic arm with Arm I - AdM and Arm I - CvM (Fig. 8) are shown for NcM at maximum reach. For this method of measurement, the base frame of the 3D-measuring machine was used only to fix the position of the robotic arm while maintaining a constant position of the base frame of the robotic arm. At the same time, the base frame of the 3D-measuring machine was used to place and fix the proximity sensor holder.

\subsection{Measurement procedure and evaluation of deviations in the position of the robotic arm}

The robotic arm was at a maximum reach of $609 \mathrm{~mm}$, according to Fig. 1, programmed by the learning method for Arm I - AdM and Arm I - CvM. For CoM and NcM, the same program was used in the robotic arm control system. The position of the proximity sensor was always adjusted to each scanned position of the robotic arm in the corresponding coordinate system. The proposed Procedure for measuring and evaluation robotic arm's position deviation is shown in Fig. 9.

\section{Theory/calculation}

The generated Ishikawa diagram (Fig. 10) defines, investigates and detects the effects of several influences and causes, which result in the variability of the robotic arm position. The expected sources of variability in deviations of the robotic arm position that affect it are:

- Arm I production technology,

- weight size.

The source of variability, which is the subject of the experiment, is the accuracy of the position of the robotic arm with respect to the used production technology. However, in addition to expected resources, other factors also affect the accuracy of the robotic arm position. The causes are divided into categories and represent potential sources of variability for measured position deviations in the robotic arm. 
Other sources of variability are undesirable in the experiment and require correction using the following methods:

- elimination - the conditions of the experiment will ensure that this source of variability does not occur in the experiment at all,

- minimization - targeted reduction of variability so that the rest will be part of the experimental error,

- part of the experimental error - we know about this source, it is impossible to treat, thus will be reflected in a random error in the calculations.

As part of ensuring the conditions for carrying out the experiment, each known source of variability was corrected accordingly.

The measuring apparatus for measuring of robotic arm's positioning accuracy by NcM, using a proximity sensor, consisted of a voltage source, connecting electrical cables, an A/D converter and a proximity sensor. The measuring apparatus for measuring of robotic arm's positioning accuracy by CoM consisted of a 3D-measuring machine RAPID and a transducer with a complete connection of sensors.

\subsection{Statistical evaluation of measurement results}

Statistical evaluation of measurement results was performed for both Arm I - AdM and Arm I - CvM with the use of CoM and NcM. In each case, 11 measurements described in Chap. 2.5 were performed, while each group of 11 measurements formed one set. The results were processed according to Fig. 9 as follows:

\section{(a) Verification that the selection of measured values comes from a population with a normal distribution.}

-Check outlier method of comparing the distance of the minimum and maximum from the first and third quartiles.

-Implementation of the Grubbs test for outlier and removal of 1 to 3 extreme values to maintain the required 8 values, as in the following tests the required number of required values is $>7$.

-Verified groups of measurements come from a population with a normal distribution performed by the Shapiro-Wilk normality test. If the hypothesis was rejected, the Kolmogorov-Smirnov normality test or the Pearson chi-square normality test was performed. If the hypothesis was rejected in all cases, then the measurements do not come from a population with a normal distribution. Otherwise, we do not reject the hypothesis of a normal distribution.

(b) Descriptive statistics.

(c) Comparison of measurement pairs. 
For Arm I - AdM, Arm I - CvM, where the other mode settings were the same, the Two Sample t-test was used for the null hypothesis (difference in means is equal to 0 ) $\mu_{-} 1=\mu_{-} 2$. To calculate the test statistic $t$, it was necessary to verify whether var.equal applies. This was preceded by an $\mathrm{F}$ test to compare two variances with the ratio of variances equal to 1 .

\section{Results And Discussion}

\subsection{Verification that the selection of measured values comes from a population with a normal distribution}

In this part, it was necessary to verify the hypothesis of a normal population distribution. The hypothesis of a normal population distribution (Table 3 ) was not rejected, i.e. the measurements were performed correctly, and the results of measurements can be further used for statistical evaluation using parametric tests.

Table 3 and Table 4 present the results for groups of measurements without outliers. 
Table 3

CoM normality test

\begin{tabular}{|c|c|c|}
\hline measurement & Shapiro-Wilk statistics & normal distribution result \\
\hline AdM_CoM_0_Xx & $\mathrm{W}=0.8942, \mathrm{p}$-value $=0.1568$ & not reject \\
\hline AdM_CoM_0_Yy & $W=0.92155, p-$ value $=0.3317$ & not reject \\
\hline AdM_CoM_0_Zz & $\mathrm{W}=0.94601, \mathrm{p}$-value $=0.5935$ & not reject \\
\hline AdM_CoM_1_Xx & $W=0.9363, p-$ value $=0.478$ & not reject \\
\hline AdM_CoM_1_Yy & $W=0.95938, p-$ value $=0.7639$ & not reject \\
\hline AdM_CoM_1_Zz & $W=0.88907, p$-value $=0.1354$ & not reject \\
\hline AdM_CoM_2_Xx & $W=0.86257, p$-value $=0.06217$ & not reject \\
\hline AdM_CoM_2_Yy & $W=0.90804, p$-value $=0.2312$ & not reject \\
\hline AdM_CoM_2_Zz & $W=0.91918, p-$ value $=0.3119$ & not reject \\
\hline CvM_CoM_0_Xx & $W=0.87682, p-$ value $=0.0948$ & not reject \\
\hline CvM_CoM_O_Yy & $W=0.96168, p-$ value $=0.7925$ & not reject \\
\hline CvM_CoM_O_Zz & $\mathrm{W}=0.92054, \mathrm{p}$-value $=0.3232$ & not reject \\
\hline CvM_CoM_1_Xx & $W=0.87293, p$-value $=0.08454$ & not reject \\
\hline CvM_CoM_1_Yy & $W=0.93015, p$-value $=0.4123$ & not reject \\
\hline CvM_CoM_1_Zz & $\mathrm{W}=0.92542, \mathrm{p}$-value $=0.3664$ & not reject \\
\hline CvM_CoM_2_Xx & $W=0.95298, p-$ value $=0.6823$ & not reject \\
\hline CvM_CoM_2_Yy & $\mathrm{W}=0.96823, \mathrm{p}$-value $=0.8682$ & not reject \\
\hline CvM_CoM_2_Zz & $\mathrm{W}=0.9256, \mathrm{p}$-value $=0.3681$ & not reject \\
\hline
\end{tabular}

Since the comparison using non-parametric methods is not possible for the measurement results, a comparison for measurements in the direction of the $Y$ axis was not performed for the mentioned sets, they are pairs: CoM_0_Yy, CoM_1_Yy, CoM_2_Yy. Statistical conclusions show for practice that in the measurements in the $\mathrm{Y}$-axis direction, there was a dimensional anomaly during the exchange of the arms, caused by non-compliance with assembly procedures and prescribed tightening torques of screws securing the arm. 


\begin{tabular}{|c|c|c|c|}
\hline \multirow[t]{3}{*}{ measurement } & \multicolumn{3}{|l|}{ statistics } \\
\hline & \multirow[t]{2}{*}{ Shapiro-Wilk } & Kolmogorov-Smirnov/ & \multirow{2}{*}{$\begin{array}{l}\text { normal } \\
\text { distribution }\end{array}$} \\
\hline & & Pearson chi-square & \\
\hline AdM_NcM_O_X & $\begin{array}{l}W=0.85477, p-\text { value }= \\
0.04924\end{array}$ & $\begin{array}{l}D=0.21081, p-\text { value }= \\
0.1872\end{array}$ & not reject \\
\hline AdM_NcM_O_Y & $W=0.93381, p-$ value $=0.4506$ & & not reject \\
\hline AdM_NcM_0_Z & $W=0.93928, p$-value $=0.5121$ & & not reject \\
\hline AdM_NcM_1_X & $W=0.95168, p-$ value $=0.6655$ & & not reject \\
\hline AdM_NcM_1_Y & $W=0.93625, p$-value $=0.4775$ & & not reject \\
\hline AdM_NcM_1_Z & $W=0.92879, p$-value $=0.3988$ & & not reject \\
\hline AdM_NcM_2_X & $\begin{array}{l}W=0.85927, p \text {-value }= \\
0.05633\end{array}$ & & not reject \\
\hline AdM_NcM_2_Y & $W=0.90404, p$-value $=0.2069$ & & not reject \\
\hline AdM_NcM_2_Z & $\mathrm{W}=0.9122, \mathrm{p}$-value $=0.2589$ & & not reject \\
\hline CvM_NcM_0_X & $\mathrm{W}=0.9326, \mathrm{p}$-value $=0.4377$ & & not reject \\
\hline CvM_NcM_O_Y & $W=0.88651, p$-value $=0.1257$ & & not reject \\
\hline CvM_NcM_0_Z & $\begin{array}{l}W=0.86383, p \text {-value }= \\
0.06454\end{array}$ & & not reject \\
\hline CvM_NcM_1_X & $W=0.97466, p$-value $=0.9293$ & & not reject \\
\hline CvM_NcM_1_Y & $\begin{array}{l}W=0.72731, p-\text { value }= \\
0.001054\end{array}$ & $\begin{array}{l}\mathrm{D}=0.26732, \mathrm{p} \text {-value }= \\
0.06298\end{array}$ & not reject \\
\hline CvM_NcM_1_Z & $W=0.92219, p-$ value $=0.3373$ & & not reject \\
\hline CvM_NcM_2_X & $W=0.8197, p-$ value $=0.02513$ & $\begin{array}{l}D=0.24475, p-\text { value }= \\
0.09133\end{array}$ & not reject \\
\hline CvM_NcM_2_Y & $W=0.98184, p$-value $=0.9755$ & & not reject \\
\hline CvM_NcM_2_Z & $\begin{array}{l}W=0.85555, p \text {-value }= \\
0.05041\end{array}$ & & not reject \\
\hline
\end{tabular}

The hypothesis of a normal population distribution for all NcM measurements in Table 4 was not rejected, so the measurements were performed correctly, and the measurement results can be further used for statistical evaluation using parametric tests. When replacing the arms and ensuring the correct assembly procedures and the prescribed tightening torques of the screws, measurements were achieved that also met the statistical requirements. 


\subsection{Descriptive statistics}

Descriptive statistics are done separately for CoM and NcM due to different measurement methodology.

\subsubsection{CoM descriptive statistics}

Descriptive one-dimensional statistics of position and variability CoM of Arm I - AdM and Arm I - CvM are in Table 5. As follows from the CoM principle (Chap. 2.3), the results in Table 5 are given in $\mathrm{mm}$.

Table 5

CoM descriptive statistics for Arm I - AdM and Arm I - CvM

\begin{tabular}{|llllllllll|}
\hline measurement & $\mathbf{n}$ & mean & sd & median & min & max & range & skew & kurt \\
\hline AdM_CoM_0_Xx & 11 & 105.65 & 0.08 & 105.67 & 105.53 & 105.75 & 0.22 & -0.29 & -1.70 \\
\hline AdM_CoM_1_Xx & 11 & 98.78 & 0.20 & 98.79 & 98.48 & 99.06 & 0.58 & -0.15 & -1.60 \\
\hline AdM_CoM_2_Xx & 11 & 98.34 & 0.14 & 98.33 & 98.20 & 98.64 & 0.44 & 0.87 & -0.49 \\
\hline AdM_CoM_0_Yy & 11 & 256.67 & 0.05 & 256.68 & 256.60 & 256.74 & 0.13 & -0.06 & -1.76 \\
\hline AdM_CoM_1_Yy & 11 & 310.56 & 0.00 & 310.56 & 310.55 & 310.56 & 0.01 & 0.03 & -1.41 \\
\hline AdM_CoM_2_Yy & 11 & 310.24 & 0.10 & 310.26 & 310.05 & 310.39 & 0.34 & -0.28 & -0.74 \\
\hline AdM_CoM_0_Zz & 11 & 214.53 & 0.15 & 214.52 & 214.30 & 214.81 & 0.52 & 0.36 & -0.69 \\
\hline AdM_CoM_1_Zz & 11 & 238.99 & 0.09 & 238.98 & 238.88 & 239.22 & 0.34 & 0.99 & 0.21 \\
\hline AdM_CoM_2_Zz & 11 & 237.95 & 0.20 & 237.93 & 237.70 & 238.40 & 0.71 & 0.71 & -0.08 \\
\hline CvM_CoM_0_Xx & 11 & 112.84 & 0.00 & 112.84 & 112.84 & 112.84 & 0.00 & 0.17 & -1.60 \\
\hline CvM_CoM_1_Xx & 11 & 109.63 & 0.09 & 109.69 & 109.47 & 109.74 & 0.27 & -0.36 & -1.58 \\
\hline CvM_CoM_2_Xx & 11 & 109.51 & 0.18 & 109.52 & 109.28 & 109.80 & 0.52 & 0.14 & -1.48 \\
\hline CvM_CoM_0_Yy & 11 & 317.08 & 0.00 & 317.08 & 317.07 & 317.08 & 0.01 & 0.51 & -0.69 \\
\hline CvM_CoM_1_Yy & 9 & 310.54 & 0.11 & 310.57 & 310.26 & 310.60 & 0.34 & -1.93 & 2.25 \\
\hline CvM_CoM_2_Yy & 10 & 310.25 & 0.14 & 310.31 & 309.93 & 310.36 & 0.44 & -1.35 & 0.34 \\
\hline CvM_CoM_0_Zz & 11 & 265.82 & 0.16 & 265.80 & 265.60 & 266.12 & 0.52 & 0.69 & -0.73 \\
\hline CvM_CoM_1_Zz & 11 & 263.93 & 0.09 & 263.96 & 263.77 & 264.04 & 0.26 & -0.34 & -1.40 \\
\hline CvM_CoM_2_Zz & 11 & 262.46 & 0.07 & 262.46 & 262.31 & 262.56 & 0.25 & -0.43 & -0.95 \\
\hline
\end{tabular}

It is clear from Table 5 that the mean values of measurements do not have the same value. Maximum variability for expression is the standard deviation sd of $0.20 \mathrm{~mm}$. Another indicator of variability is the range. The skewness indicator describes the skewing of selections. In cases of measurements in the 
direction of the $\mathrm{Y}$ axis, when the hypothesis of a normal distribution of sets was rejected, high values of this indicator can be observed. The kurtosis indicator should be approximately 0 . Negative values less than -1 indicate that the frequency distribution curve is too flat. Positive values indicate that the point density is too high. The minimum, maximum and median are shown by the box graphs in Fig. 11-13.

For measurements in the direction of the X axis (Fig. 11), for Arm I - AdM and Arm I - CvM, a significant decrease in the median position of the arm showed when changing the load from $0 \mathrm{~kg}$ to $1 \mathrm{~kg}$ and a slight decrease in median position is still visible when changing the load from $1 \mathrm{~kg}$ to $2 \mathrm{~kg}$. The variability of measurements in the direction of the $\mathrm{X}$ axis evaluated by range and standard deviation sd is highest for the measurement AdM_CoM_1_Xx. With both types of arms, the robot arm decreases in the X-axis direction as the load increases.

For the measurement in the direction of the $Y$ axis (Fig. 12), for Arm I - AdM, there is a significant increase in the median position when changing the load from $0 \mathrm{~kg}$ to $1 \mathrm{~kg}$ and a slight decrease in the median position when changing the load from $1 \mathrm{~kg}$ to $2 \mathrm{~kg}$. Arm I - CvM shows a significant decrease in the median position when changing the load from $0 \mathrm{~kg}$ to $1 \mathrm{~kg}$, and a slight decrease in the median position when changing the load from $1 \mathrm{~kg}$ to $2 \mathrm{~kg}$. The highest range is for measurements in the direction of the $Y$ axis for CvM_CoM_2_Yy, namely range $0.44 \mathrm{~mm}$ and standard deviation sd of $0.14 \mathrm{~mm}$.

For measurements in the direction of the $Z$ axis (Fig. 13), for Arm I - AdM, there is a significant increase in the median position when changing the load from $0 \mathrm{~kg}$ to $1 \mathrm{~kg}$ and slightly decreasing in the median position when changing the load from $1 \mathrm{~kg}$ to $2 \mathrm{~kg}$. Arm I - CvM shows a slight decrease in the median position when changing the load from $0 \mathrm{~kg}$ to $1 \mathrm{~kg}$ and a slight decrease in the median position when changing the load from $1 \mathrm{~kg}$ to $2 \mathrm{~kg}$. The range in the direction of the $Z$ axis is the largest for measurement AdM_CoM_2_Zz, namely the range up to $0.71 \mathrm{~mm}$ and the standard deviation sd of $0.20 \mathrm{~mm}$. Figure $11-$ 13 show that both Arm I - AdM and Arm I - CvM, without load, will reach a different position than when measuring with load. The resulting increase in the position of the robotic arm in the $Z$ axis direction using Arm I - AdM with respect to Arm I - CvM, is caused by springing of the Arm I - AdM structure.

\subsubsection{NcM descriptive statistics}

Descriptive one - dimensional NcM position and variability statistics for Arm I - AdM and Arm I - CvM are in Table 6. As follows from the CoM principle (Chap. 2.3), the results in Table 5 are given in $\mathrm{mm}$. 
Table 6

Descriptive statistics NcM for Arm I - AdM and Arm I - CvM

\begin{tabular}{|lllllllllc|}
\hline measurements & $\mathbf{n}$ & $\begin{array}{c}\text { mean } \\
{[\boldsymbol{\mu m}]}\end{array}$ & $\begin{array}{l}\mathbf{s d} \\
{[\boldsymbol{\mu m}]}\end{array}$ & $\begin{array}{c}\text { median } \\
{[\mu \mathrm{m}]}\end{array}$ & $\begin{array}{c}\text { min } \\
{[\boldsymbol{\mu m}]}\end{array}$ & $\begin{array}{c}\text { max } \\
{[\boldsymbol{\mu m}]}\end{array}$ & $\begin{array}{c}\text { range } \\
{[\mu \mathrm{m}]}\end{array}$ & skewness & kurtosis \\
\hline AdM_NcM_0_X & 11 & 212.64 & 16.76 & 208 & 196 & 240 & 44 & 0.51 & -1.55 \\
\hline AdM_NcM_1_X & 11 & 267.64 & 10.00 & 267 & 251 & 283 & 32 & 0.16 & -1.21 \\
\hline AdM_NcM_2_X & 11 & 295.27 & 68.40 & 262 & 218 & 390 & 172 & 0.22 & -1.86 \\
\hline AdM_NcM_0_Y & 11 & 342.36 & 13.32 & 347 & 321 & 362 & 41 & -0.17 & -1.59 \\
\hline AdM_NcM_1_Y & 11 & 424.45 & 12.75 & 429 & 401 & 440 & 39 & -0.40 & -1.27 \\
\hline AdM_NcM_2_Y & 11 & 468.09 & 20.59 & 470 & 438 & 493 & 55 & -0.13 & -1.78 \\
\hline AdM_NcM_0_Z & 11 & 332.55 & 58.75 & 350 & 250 & 425 & 175 & 0.10 & -1.42 \\
\hline AdM_NcM_1_Z & 11 & 253.27 & 37.84 & 250 & 175 & 330 & 155 & -0.02 & 0.25 \\
\hline AdM_NcM_2_Z & 11 & 150.73 & 26.60 & 155 & 110 & 185 & 75 & -0.17 & -1.76 \\
\hline CvM_NcM_0_X & 11 & 425.00 & 5.81 & 425 & 416 & 433 & 17 & -0.23 & -1.58 \\
\hline CvM_NcM_1_X & 11 & 276.36 & 3.01 & 276 & 272 & 282 & 10 & 0.32 & -1.08 \\
\hline CvM_NcM_2_X & 10 & 260.80 & 0.79 & 261 & 260 & 262 & 2 & 0.29 & -1.50 \\
\hline CvM_NcM_0_Y & 11 & 207.91 & 7.57 & 207 & 200 & 225 & 25 & 0.86 & -0.30 \\
\hline CvM_NcM_1_Y & 9 & 619.22 & 20.58 & 629 & 568 & 632 & 64 & -1.63 & 1.33 \\
\hline CvM_NcM_2_Y & 11 & 593.27 & 29.18 & 600 & 542 & 638 & 96 & -0.17 & -1.27 \\
\hline CvM_NcM_0_Z & 11 & 443.18 & 44.35 & 462 & 339 & 492 & 153 & -1.07 & 0.07 \\
\hline CvM_NcM_1_Z & 11 & 135.73 & 13.38 & 132 & 112 & 153 & 41 & -0.07 & -1.36 \\
\hline CvM_NcM_2_Z & 11 & 346.00 & 48.34 & 350 & 289 & 409 & 120 & -0.10 & -1.86 \\
\hline
\end{tabular}

From Table 6 and Fig. 14, for Arm I - AdM measurements in the direction of the X axis, it is possible to observe a higher value of mean and lower range and standard deviation sd when the load is increased to $1 \mathrm{~kg}$, range and standard deviation sd increased when the load was increased to $2 \mathrm{~kg}$ and the median has shifted. For Arm I - CvM in the direction of the $\mathrm{X}$ axis, it is possible to observe a decrease in the median value as well as the mean when the load increases, and at the same time a decrease of range and standard deviation sd. It follows from the above behavior of the position of the robotic arm Arm I - AdM and Arm I - CvM and is due to the rigidity of the sensor holder.

From Table 6 and Fig. 15, for Arm I - AdM, measurements in the $Y$ - axis direction, an increase in the median value can be observed as the load increases and the range and standard deviation sd change slightly. For Arm I - CvM in the direction of the $Y$ axis, it is possible to observe an increase in the median 
and the mean with increasing load, while the range and standard deviation sd is higher at higher loads. Failure to meet the condition of normal distribution of measured values is also confirmed by anomalies in Fig. 15, at the same time, the stress caused by the construction of Arm I-AdM needs to be considered.

From Table 6 and Fig. 16, for Arm I - AdM measurements in the direction of the $Z$ axis, it can be observed that the median value always decreased and the range changed slightly due to the increase in load. For Arm I - CvM in the direction of the $Z$ axis, it is possible to observe that the median value and the mean oscillate with increasing load and the range is not always the same. The expected course, met by Arm IAdM, is caused by the interruption of the Arm I - AdM suspension, and at the same time suspension of the sensor holder. The increase in Arm I - CvM at $2 \mathrm{~kg}$ load was again caused only by the springing of the sensor holder.

Presented results indicate a statistical significance of differences in positions of the robotic arm endpoint for Arm I - AdM and Arm I - CvM, in position and variability. Since values in the table and the graph are in micrometers, these differences are negligible from a practical point of view. For further investigation, it would be appropriate to plan experimental measurements so that to better examine whether the differences are random or caused by a factor that has not yet been considered. This was affected by: arm flexibility, loading time, movement time of the robotic arm, sensitivity of the proximity sensor and rigidity of the sensor holder.

Due to the fact that the production documentation of Arm I - AdM and Arm I - CvM contained dimensional tolerances in micrometers and was observed, the position shift could occur during the mutual exchange disassembly and assembly of Arm I - AdM for Arm I - CvM.

\subsubsection{Comparison of Arm I - AdM and Arm I - CvM measurement pairs}

Table 7 shows the results of the $\mathrm{F}$ test and the $\mathrm{t}$ test for CoM. It is clear from Table 7 that no match of the mean values is confirmed for any pair. In four cases, for the CoM_0_Zz, CoM_1_Zz, CoM_2_Xx and CoM_2_Yy modes, the agreement of the variances is confirmed. 
Table 7

CoM $F$ test and $t$ test

\begin{tabular}{|lllllll|}
\hline measurements & F test statistics & p-value & result & $\mathbf{t}$ test statistics & p value & result \\
\hline CoM_0_Xx & 0.00050691 & $8.399 \mathrm{e}-15$ & reject & 290.89 & $<2.2 \mathrm{e}-16$ & reject \\
\hline CoM_0_Yy & 0.0044277 & $4.133 \mathrm{e}-10$ & reject & 4281 & $<2.2 \mathrm{e}-16$ & reject \\
\hline CoM_0_Zz & 1.1331 & 0.8472 & not reject & 793.81 & $<2.2 \mathrm{e}-16$ & reject \\
\hline CoM_1_Xx & 0.21501 & 0.02322 & reject & 163.92 & $<2.2 \mathrm{e}-16$ & reject \\
\hline CoM_1_Yy & 0.0099339 & $2.245 \mathrm{e}-08$ & reject & 164.76 & $<2.2 \mathrm{e}-16$ & reject \\
\hline CoM_1_Zz & 0.90569 & 0.8786 & not reject & 633.53 & $<2.2 \mathrm{e}-16$ & reject \\
\hline CoM_2_Xx & 1.6025 & 0.4691 & not reject & 163.31 & $<2.2 \mathrm{e}-16$ & reject \\
\hline CoM_2_Yy & 0.49794 & 0.2869 & not reject & 625.65 & $<2.2 \mathrm{e}-16$ & reject \\
\hline CoM_2_Zz & 0.13712 & 0.004215 & reject & 382.36 & $<2.2 \mathrm{e}-16$ & reject \\
\hline
\end{tabular}

Table 8 shows the results of the $\mathrm{F}$ test and the $\mathrm{t}$ test for $\mathrm{NcM}$.

Table 8

NcM F test and t test

\begin{tabular}{|c|c|c|c|c|c|c|}
\hline measurements & $\begin{array}{l}\text { F test } \\
\text { statistics }\end{array}$ & p-value & result & $\begin{array}{l}t \text { test } \\
\text { statistics }\end{array}$ & $p$ value & result \\
\hline NcM_0_X & 0.12035 & 0.002481 & reject & 39.706 & $\begin{array}{l}2.012 \mathrm{e}- \\
14\end{array}$ & reject \\
\hline NcM_0_Y & 0.32285 & 0.08889 & $\begin{array}{l}\text { not } \\
\text { reject }\end{array}$ & -29.105 & $<2.2 \mathrm{e}-16$ & reject \\
\hline NcM_0_Z & 0.57004 & 0.3891 & $\begin{array}{l}\text { not } \\
\text { reject }\end{array}$ & 4.9848 & $\begin{array}{l}7.117 \mathrm{e}- \\
05\end{array}$ & reject \\
\hline NcM_1_X & 0.090496 & 0.0007453 & reject & 2.7711 & 0.01717 & reject \\
\hline NcM_1_Y & 2.603 & 0.1582 & $\begin{array}{l}\text { not } \\
\text { reject }\end{array}$ & 25.963 & $\begin{array}{l}1.022 \mathrm{e}- \\
15\end{array}$ & reject \\
\hline NcM_1_Z & 0.12503 & 0.002901 & reject & -9.7135 & $\begin{array}{l}3.587 e- \\
07\end{array}$ & reject \\
\hline NcM_2_X & 0.00013299 & $4.261 \mathrm{e}-16$ & reject & -1.6714 & 0.1256 & $\begin{array}{l}\text { not } \\
\text { reject }\end{array}$ \\
\hline NcM_2_Y & 2.0091 & 0.2866 & $\begin{array}{l}\text { not } \\
\text { reject }\end{array}$ & 11.625 & $\begin{array}{l}2.377 e^{-} \\
10\end{array}$ & reject \\
\hline NcM_2_Z & 3.08 & 0.0731 & $\begin{array}{l}\text { not } \\
\text { reject }\end{array}$ & 11.738 & $\begin{array}{l}2.006 \mathrm{e}- \\
10\end{array}$ & reject \\
\hline
\end{tabular}


It is clear from Table 8 that no agreement of the mean values is confirmed for any pair. In six cases, for NcM_0_Y, NcM_0_Z, NcM_1_Y, NcM_2_Y, NcM_2_Z, and NcM_2_X modes, the variance agreement is confirmed. The test results in Table 7 and Table 8 confirm that Arm I - AdM and Arm I - CvM are not interchangeable in this study.

\section{Conclusions}

The proposed methodology for measuring of robotic arm positioning accuracy was verified on its construction consisting of three arms Arm I, II, III, while Arm I was manufactured by two different technologies AdM and CvM. A series of repeated measurements was performed for each robotic arm configuration with two different measurement methods CoM and NcM. The results of measurements were verified by statistical methods, based on which unsatisfactory values of measurements were excluded from the evaluation.

The use of proposed methodology is not only in the field of metrology and testing, but also to verify the interchangeability of components in construction of robotic systems. The methodology provides a detailed view of a dimensional chain quality of the robotic arm structure and determines conditions for maintaining accuracy during disassembly and reassembly of individual components of robotic system structures.

Statistical evaluation of the results verified that the obtained data was measured correctly and have a normal distribution.

For CoM with the same load of Arm I - AdM and Arm I - CvM:

- homoscedasticity was confirmed for: CoM_0_Zz, CoM_1_Zz, CoM_2_Xx and CoM_2_Yy,

- the conformity of the mean values has not been confirmed.

This means that for interchangeable Arm I it is necessary to modify the design of ArM I - AdM,

For NcM with the same load of Arm I - AdM and Arm I - CvM:

- homoscedasticity was confirmed for: NcM_0_Y, NcM_1_Y, NcM_2_Y, NcM_0_Z, NcM_2_Z,

- the conformity of the mean values for NcM_2_X has been confirmed.

This means that for mutually interchangeable Arm I - AdM, Arm I - CvM, to increase its rigidity, the structural design of the sensor holder must be modified.

Due to confirmed differences in positions for the same measured axis in different types of arms, we assume that Arm I - AdM, Arm I - CvM or an arm made by different technology may have dimensional deviations. From previous observations and calculations, for the research of other factors influencing the localization of the robotic arm position, these deviations in dimensions need to be eliminated. 
Based on the results, we can conclude that production technology does not affect the positioning accuracy of the robotic arm, but the design of Arm I - AdM needs to be changed regardless of the operating load.

To further investigate, it will be appropriate to plan experimental measurements to better examine whether differences in a position of the robot arm are random or caused by loads or other factors that have not been taken into account (arm flexibility, load time, transport time, programmed trajectory of the robot arm...). The answer to the question will be known after analysis of accuracy of measured positions for Arm I - AdM and Arm I - CvM, depending on the measurement directions (X, Y, Z) and the load.

In the future, to increase the accuracy of measurement process, it will be necessary to use the proximity sensor MTN/EP080 Probe with higher sensitivity and increase rigidity of the proximity sensor holder. Furthermore, ensure the same position of the sensor holder for Arm I - AdM, Arm I - CvM on the robotic arm.

\section{Abbreviations}

AdM made by additive manufacturing

CvM made by CNC milling

CoM Contact measuring Method

D test statistics test statistics Kolmogorov-Smirnov test

max maximum

min minimum

NcM Non-contact measuring Method

$p$ value probability that the null hypothesis is true

P test statistics Pearson chi-square test

sd standard deviation

W test statistics Shapiro-Wilk test

XxX_YyY_L_A measurement regime with marking:

XxX - Manufacturing technology, [AdM or CvM]

YyY - Measuring method, [CoM or NcM]

$\mathrm{L}$ - load [0 kg or $1 \mathrm{~kg}$ or $2 \mathrm{~kg}$ ] 
A - measuring axis $[\mathrm{X}$ or $\mathrm{Y}$ or $\mathrm{Z}]$

Marking examples:

AdM_CoM_0_Xx "made by additive manufacturing, CoM measurement, load 0 kg, measuring axis X"

CvM_CoM_2_Zz "made by CNC milling, CoM measurement method, load 2kg, measuring axis Z"

AdM_NcM_0_X "made by additive manufacturing, NoC measurement method, load 0kg, measuring axis X"

CvM_NcM_2_Z "made by CNC milling, NoC measurement method, load 2kg, measuring axis Z"

\section{Declarations}

Funding: This work was supported by The Ministry of Education, Science, Research and Sport of the Slovak Republic (Grant numbers VEGA 1/0045/18, VEGA 1/0403/18, VEGA 1/0600/20, VEGA 1/0264/21), Cultural and Educational Grant Agency of the Ministry of Education, Science, Research and Sport of the Slovak Republic (Grant numbers KEGA 012TUKE-4/2019, KEGA 013TUKE-4/2019) and The Slovak Research and Development Agency (Grant number APVV SK-SRB-18-0053).

Conflicts of interest: The authors declare that they have no conflict of interest.

Availability of data and material: Not applicable

Code availability: Not applicable

Ethics approval: Not applicable

Consent to participate: Not applicable

Consent for publication: Not applicable

Authors' contributions: Not applicable

\section{References}

1. Barbosa WS, Gioia MM, Natividade VG, et al (2020) Industry 4.0: examples of the use of the robotic arm for digital manufacturing processes. Int J Interact Des Manuf 14:1569-1575 . doi: $10.1007 /$ s12008-020-00714-4

2. Angeles J (2003) Fundamentals of Robotic Mechanical Systems: Theory, Methods, and Algorithms, 2nd ed. Springer, New York, USA

3. Reza N (2010) Theory of Applied Robotics: Kinematics, Dynamics, and Control, 2nd ed. Springer US, New York, USA 
4. Covaciu F, Filip D (2019) Design and manufacturing of a 6 degree of freedom robotic arm. Acta Tech Napocensis Ser Math Mech Eng 62:107-114

5. Gordaninejad F, Vaidyaraman S (1994) Active and passive control of a revolute-prismatic flexible composite-material robot arm. Comput Struct 53:867-875 . doi: 10.1016/0045-7949(94)90375-1

6. Gibson I, Rosen DW, Stucker B (2010) Additive Manufacturing Technologies. Springer, Boston, MA, Boston

7. Ishak I, Larochelle P (2017) Robot Arm Platform for Additive Manufacturing: 3D Lattice Structures. In: 30th Florida Conference on Recent Advances in Robotics. Florida Atlantic University, Boca Raton, pp 1-5

8. Hajash K, Sparrman B, Guberan C, et al (2017) Large-scale rapid liquid printing. 3D Print Addit Manuf 4:123-131 . doi: 10.1089/3dp.2017.0037

9. Mick S, Lapeyre M, Rouanet P, et al (2019) Reachy, a 3D-printed human-like robotic arm as a testbed for human-robot control strategies. Front Neurorobot 13:1-12 . doi: 10.3389/fnbot.2019.00065

10. Costa A, Abdel-Rahman A, Jenett B, et al (2019) Algorithmic Approaches to Reconfigurable Assembly Systems. IEEE Aerosp Conf Proc 2019-March: . doi: 10.1109/AERO.2019.8741572

11. Ismail MH, Qi ANW, Voon KL, et al (2015) Design and development of a mechanism of robotic arm for lifting Part5. In: 2nd Integrated Design Project Conference (IDPC). Universiti Malaysia, Pahang, pp $1-15$

12. Wang J, Xiao X, Huang Z, Melzer A (2019) 3D-printing based Transducer Holder for Robotic Assisted Ultrasound Guided HIFU. Procedia Manuf 30:3-10 . doi: 10.1016/j.promfg.2019.02.002

13. Kluz R, Trzepieciński T (2014) The repeatability positioning analysis of the industrial robot arm. Assem Autom 34:285-295 . doi: 10.1108/AA-07-2013-070

14. Elvira-Ortiz DA, De Romero-Troncoso RJ, Jaen-Cuellar AY, et al (2016) Vibration Suppression for Improving the Estimation of Kinematic Parameters on Industrial Robots. Shock Vib 2016: . doi: $10.1155 / 2016 / 6954012$

15. Rodriguez-Donate C, Osornio-Rios RA, Rivera-Guillen JR, Romero-Troncoso R de J (2011) Fused smart sensor network for multi-axis forward kinematics estimation in industrial robots. Sensors (Switzerland) 11:4335-4357 . doi: 10.3390/s110404335

16. Joubair A, Slamani M, Bonev IA (2013) Kinematic calibration of a five-bar planar parallel robot using all working modes. Robot Comput Integr Manuf 29:15-25 . doi: 10.1016/j.rcim.2012.10.002

17. Kluz R, Kubit A, Trzepiecinski T (2018) Investigations of temperature-induced errors in positioning of an industrial robot arm. J Mech Sci Technol 32:5421-5432 . doi: 10.1007/s12206-018-1040-9

18. https://www.ge.com/additive/additive-manufacturing/machines/dmlm-machines/x-line-2000r

19. https://www.directindustry.com/prod/thome-praezision/product-24606-405507.html

20. https://amcvibro.com/product/mtn-ep080-probe/

\section{Figures}



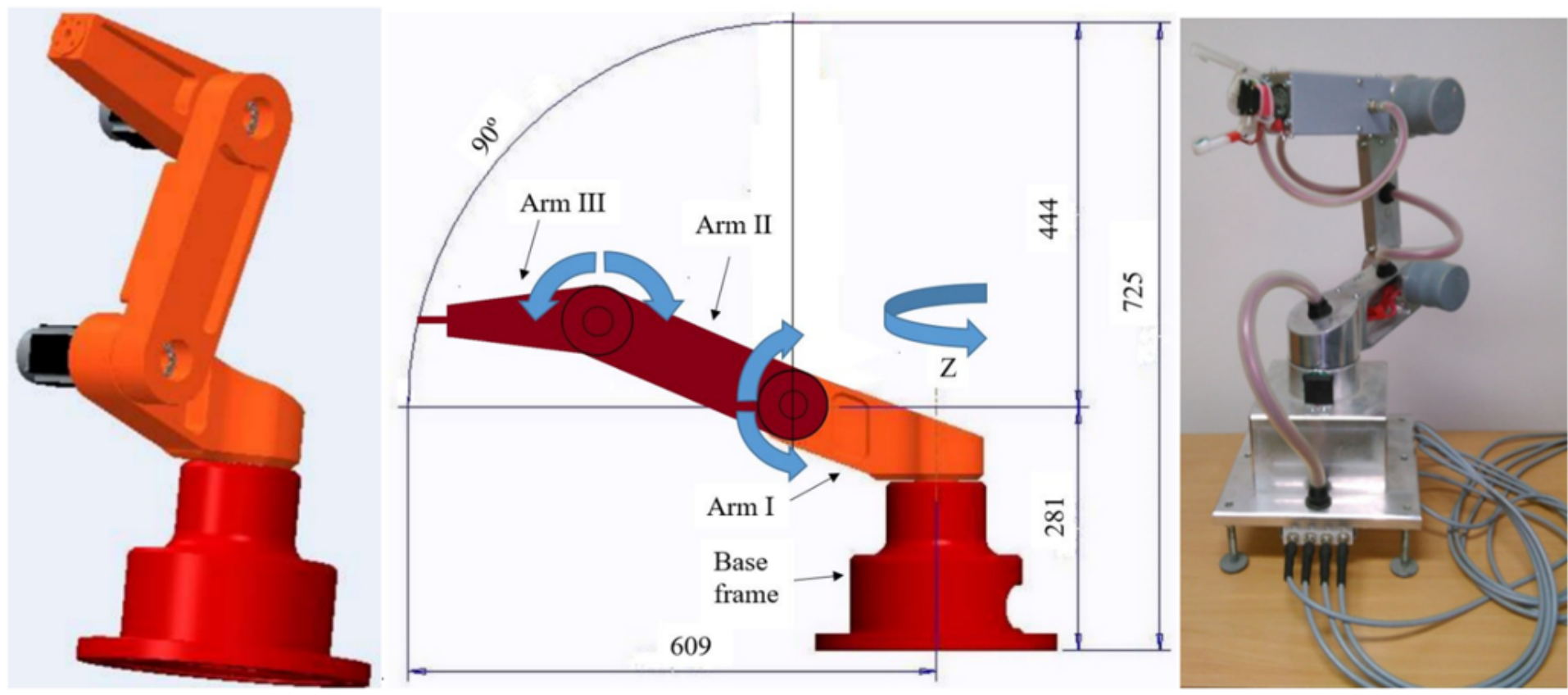

Figure 1

Robot with angular workspace (dimensions in $\mathrm{mm}$ ).
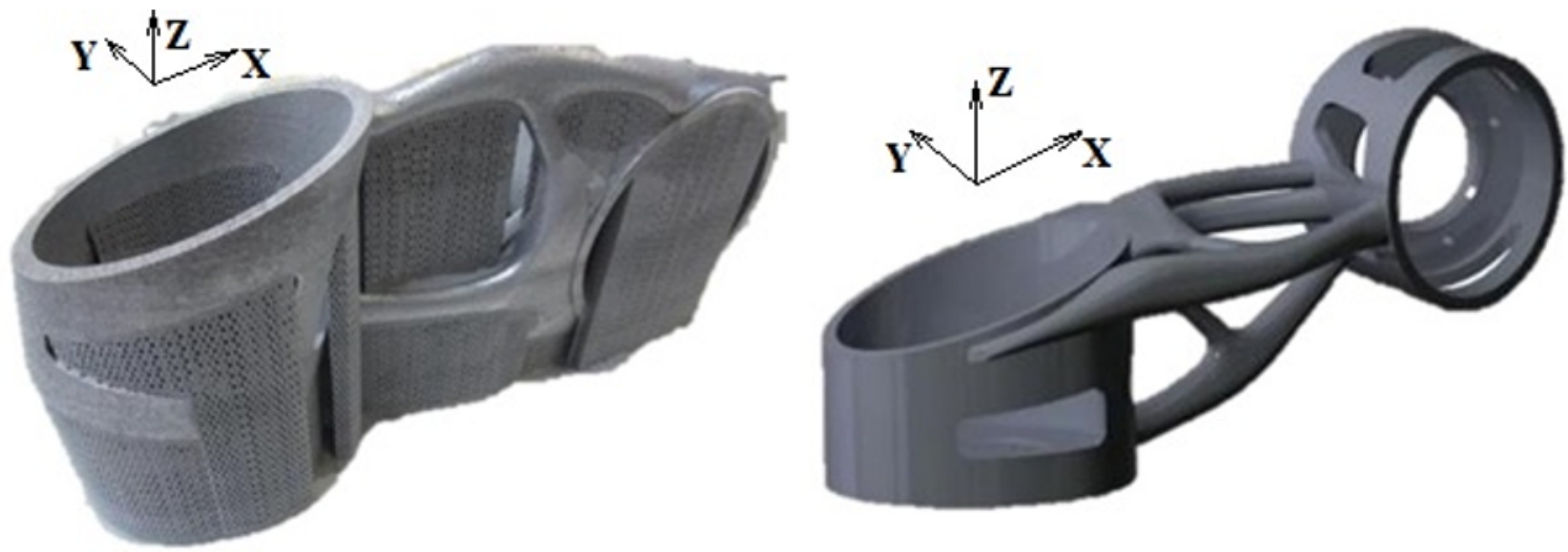

Figure 2

Arm I - AdM made on 3D printer Xline 2000R. 


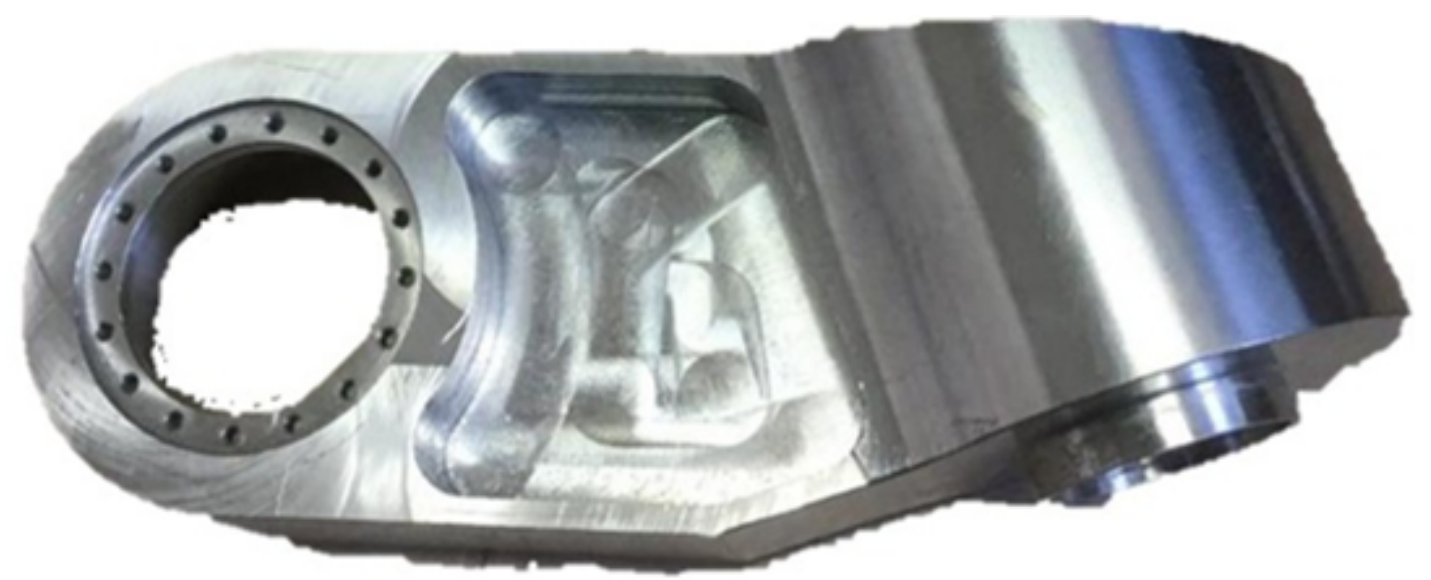

Figure 3

Arm I - CvM made on CNC machine Pinnacle VMC 650S.

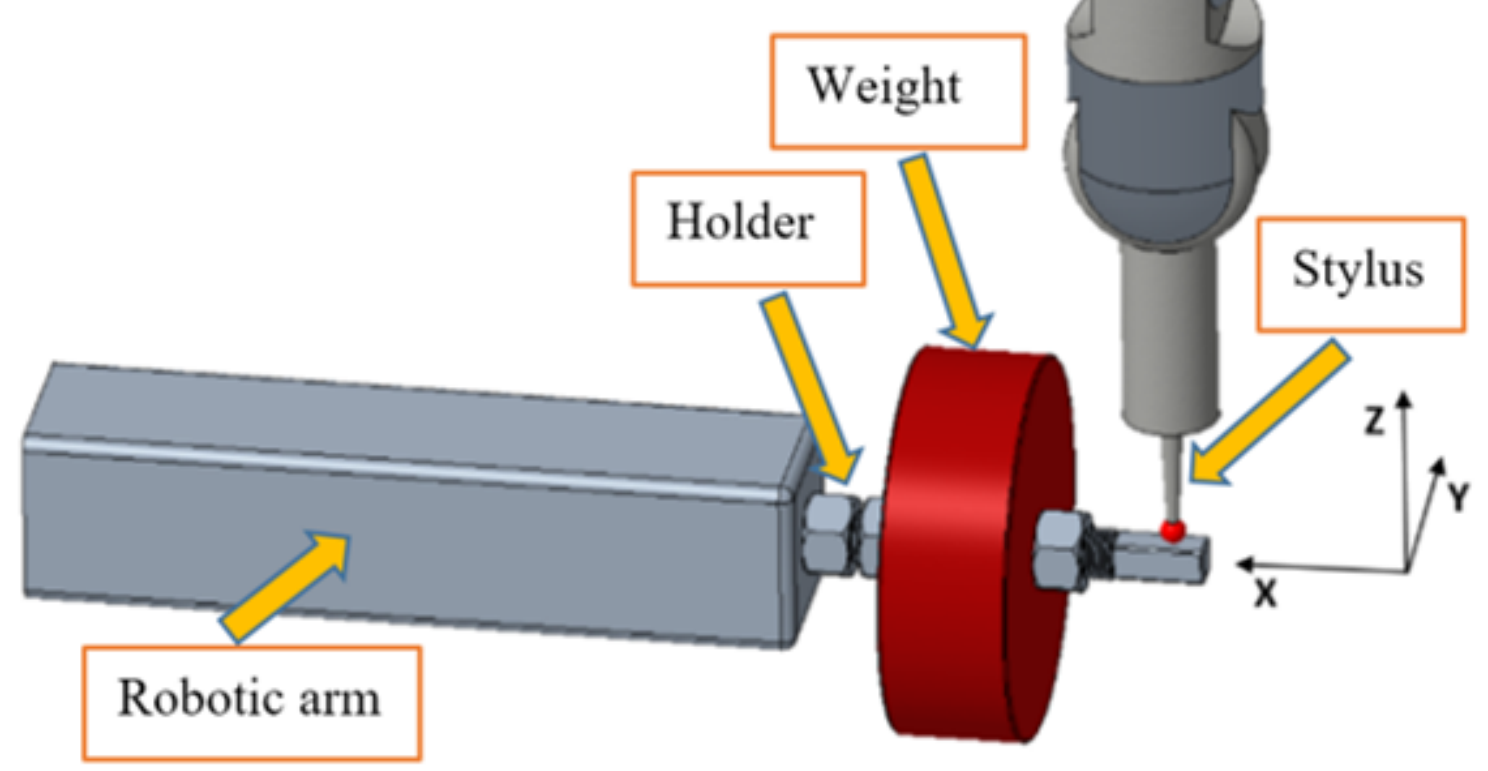

Figure 4

CoM of robotic arm's position using a 3D-measuring machine. 


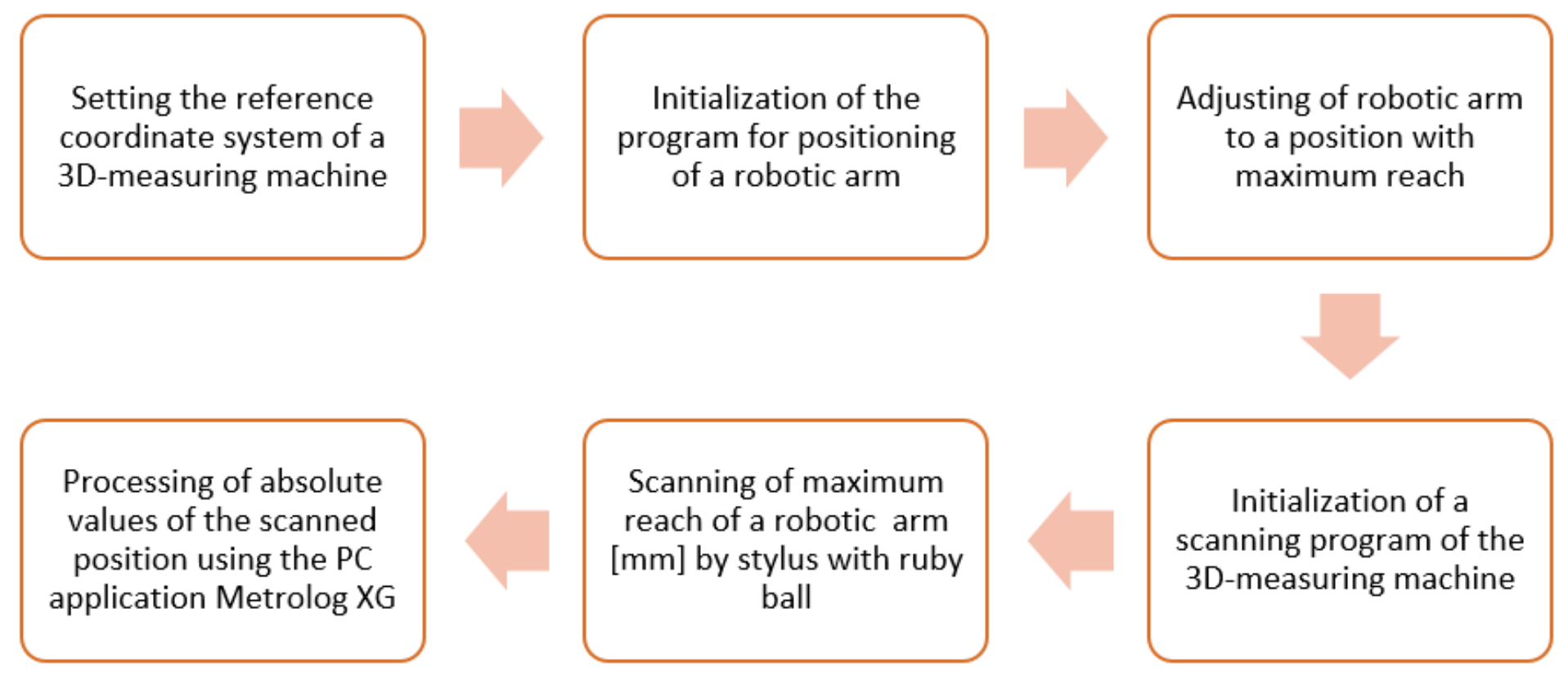

\section{Figure 5}

Measuring chain for CoM of robotic arm's position. 

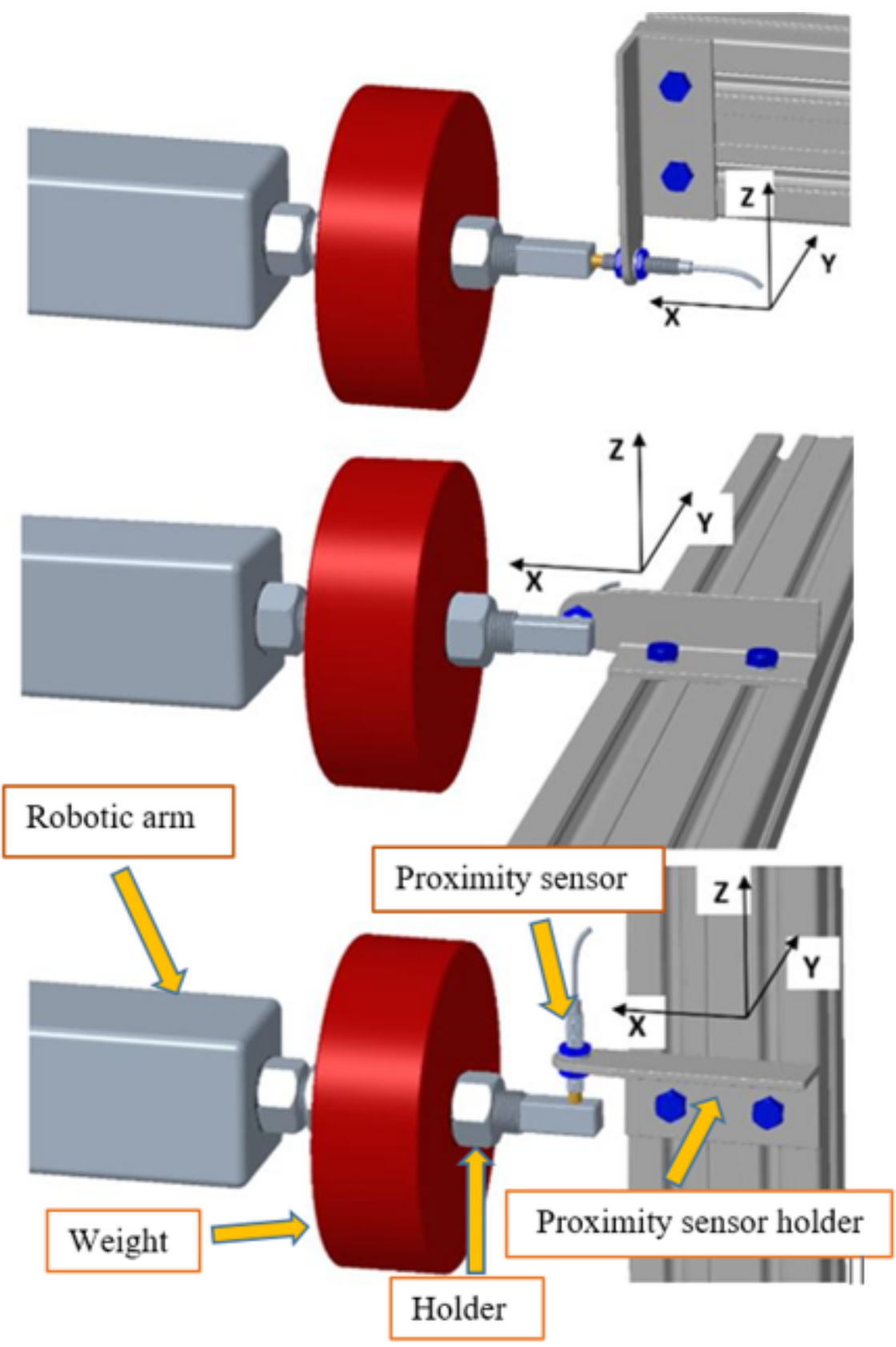

Figure 6

NcM of robotic arm's position deviation using a proximity sensor in the direction of the $X, Y, Z$ axis. 


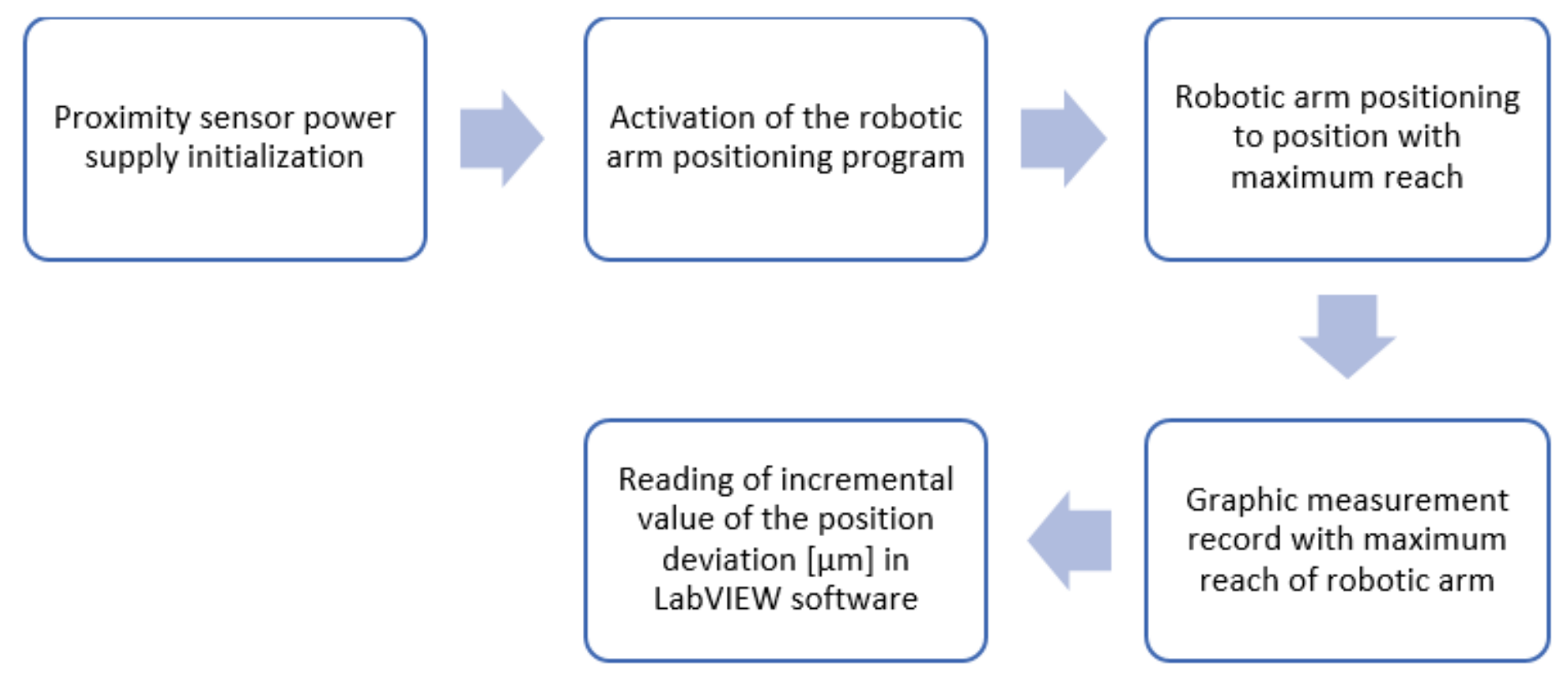

Figure 7

Measuring chain for NcM of robotic arm's position deviation.

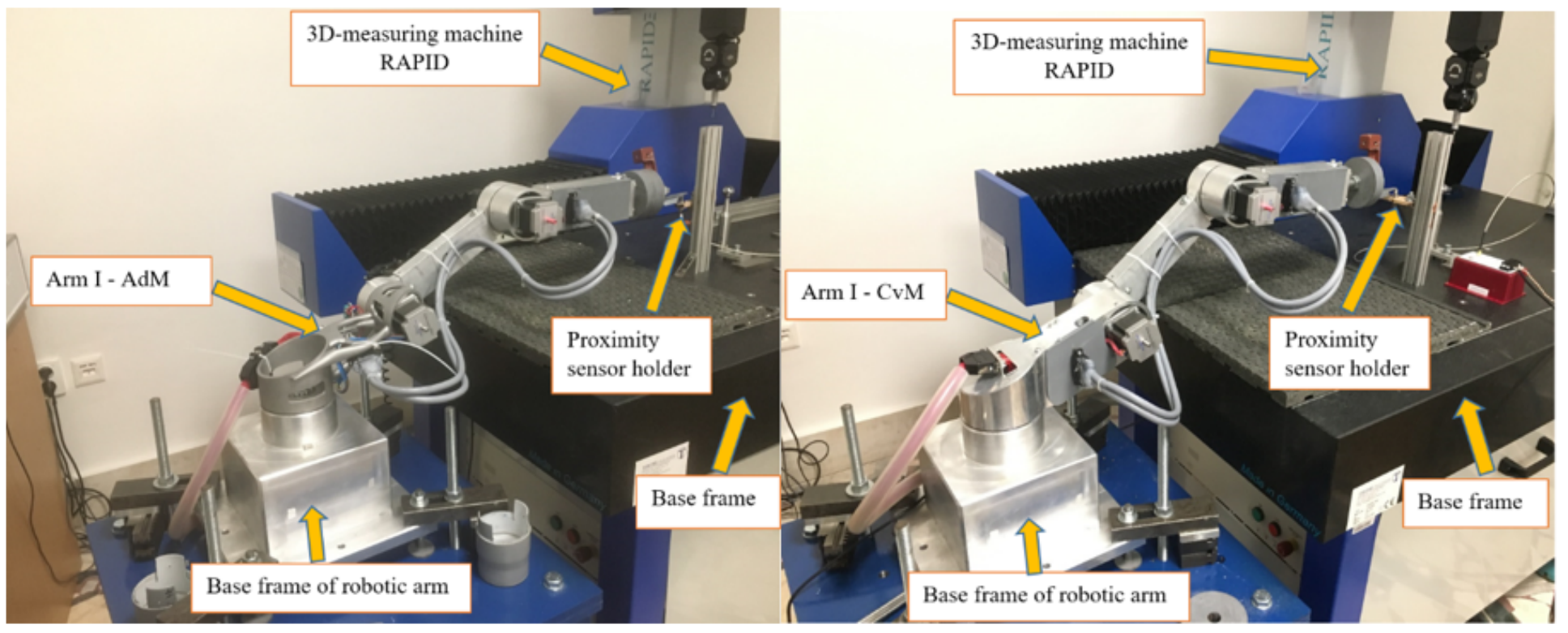

Figure 8

NcM of robotic arm's position deviation with Arm I - AdM and Arm I - CvM. 


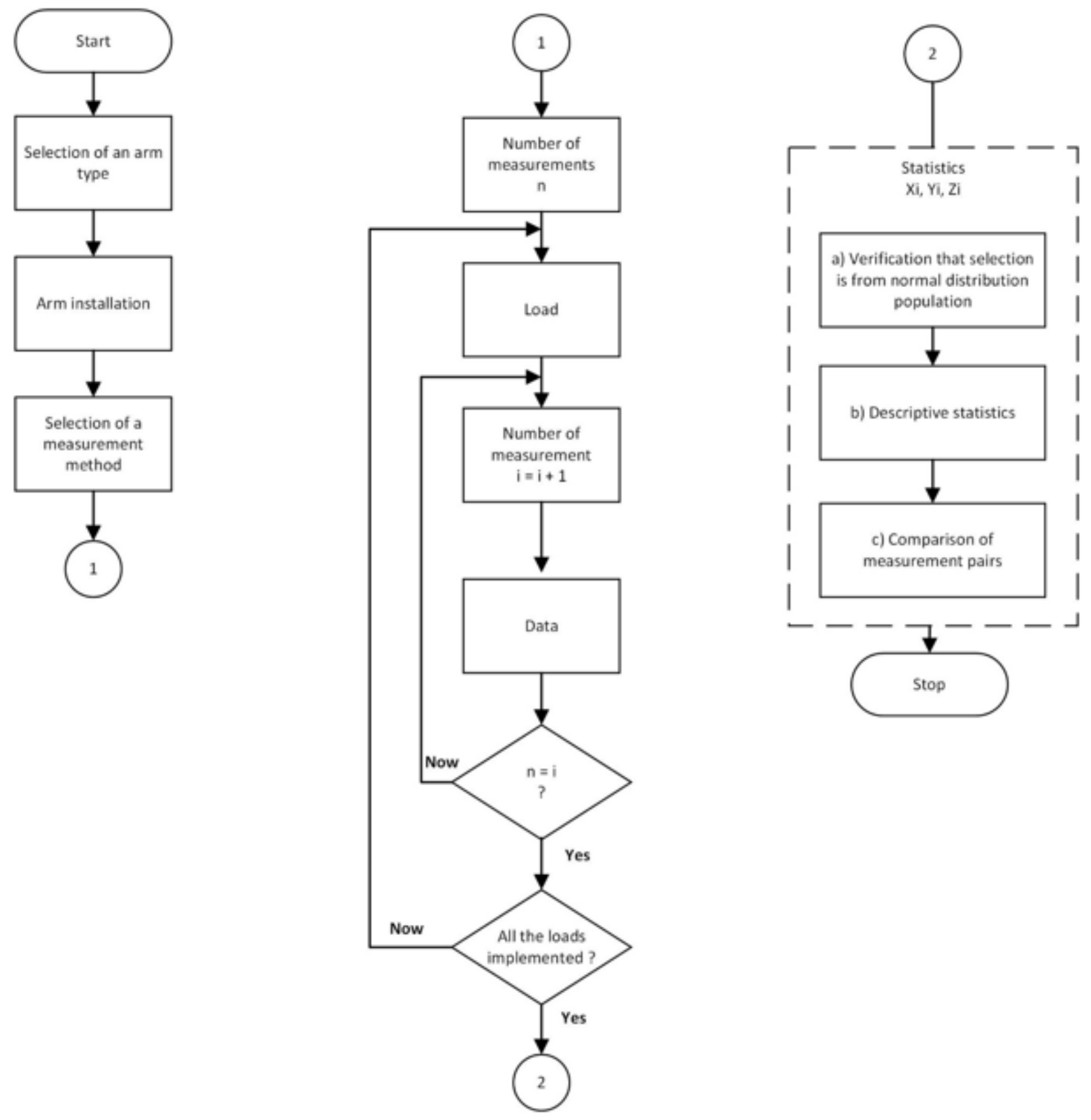

Figure 9

Procedure for measuring and evaluation of a robotic arm position deviation. 


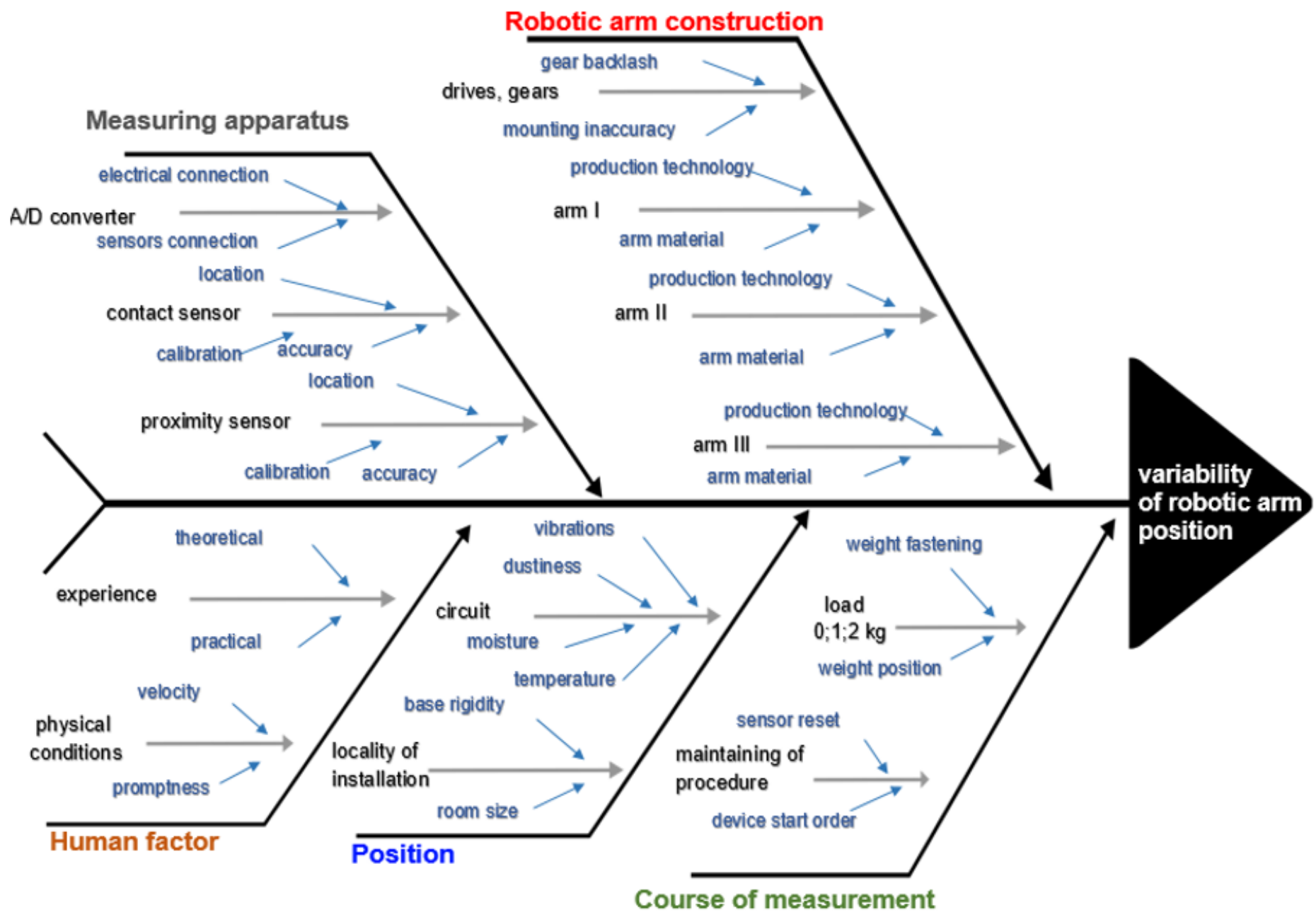

Figure 10

Causes and effects diagram. 


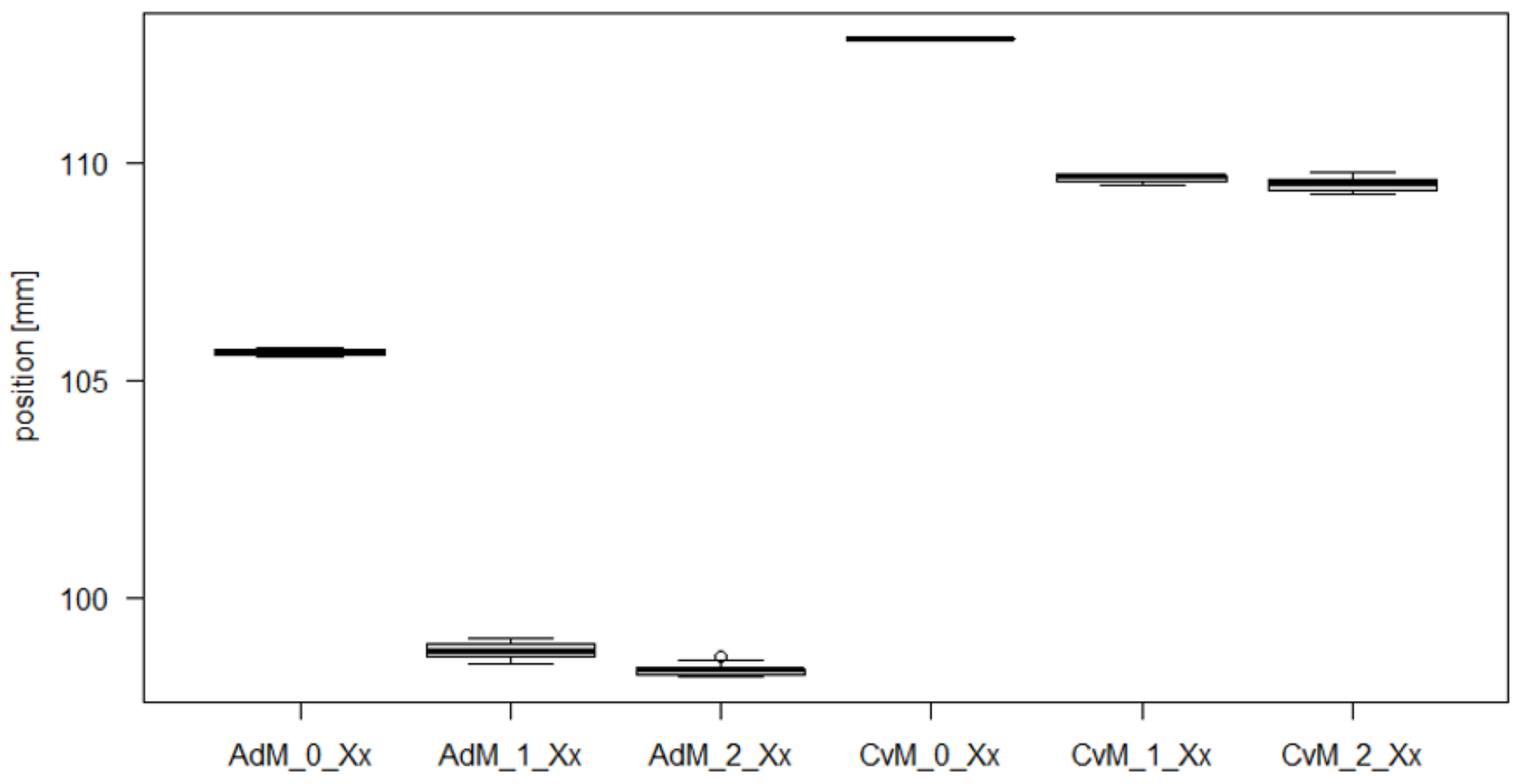

Figure 11

Measurements CoM axis $\mathrm{X}$.

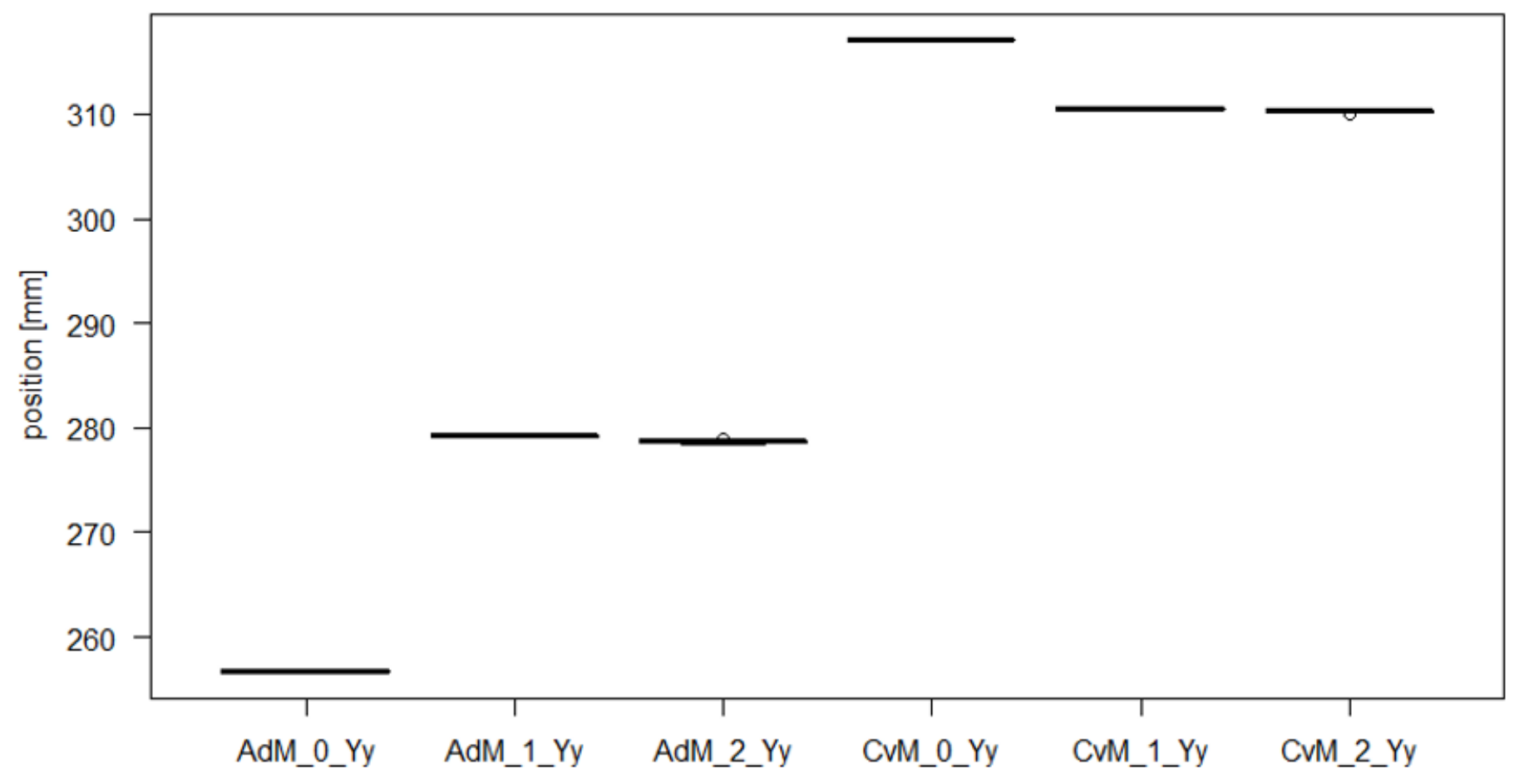

Figure 12

Measurements CoM axis Y. 


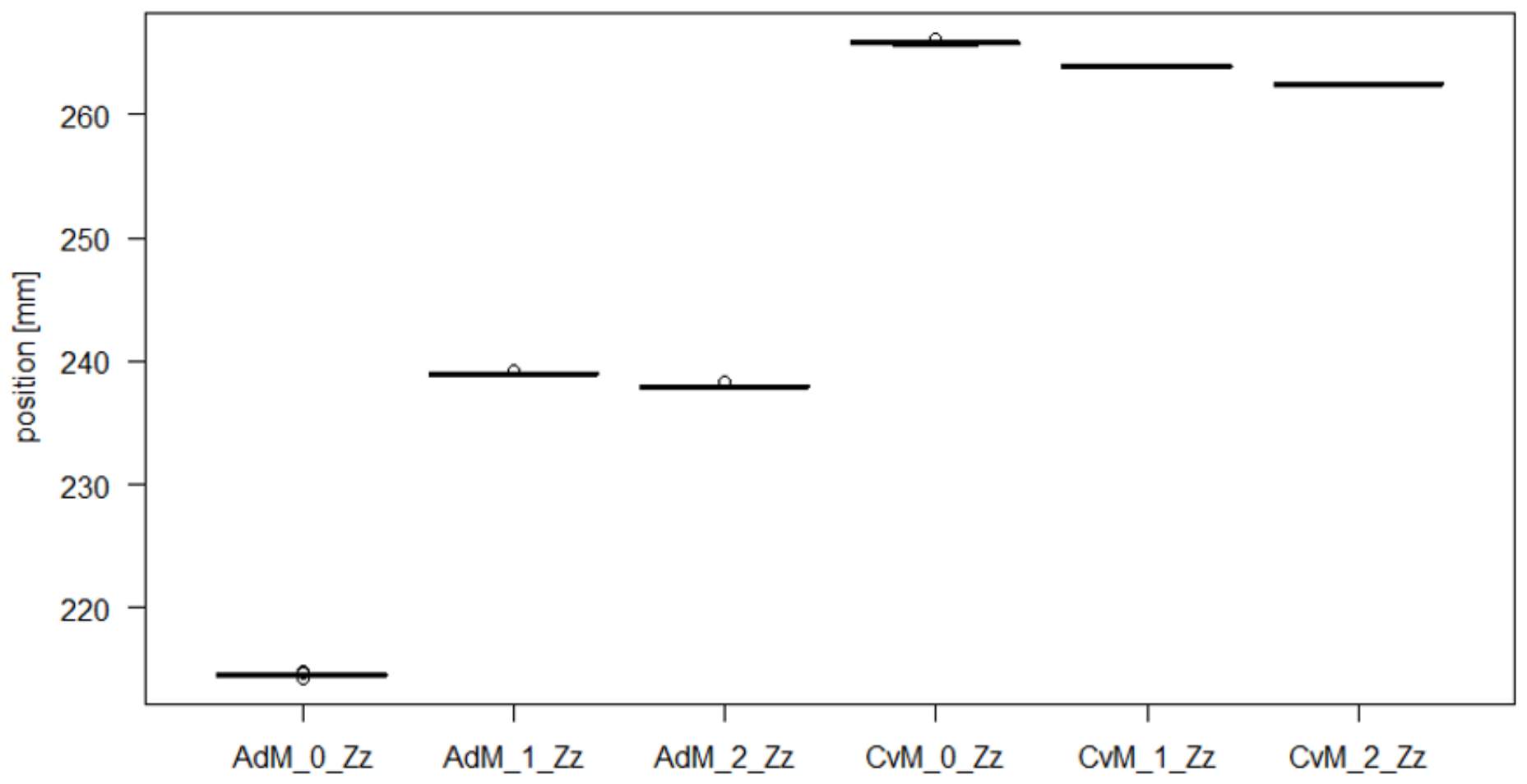

Figure 13

Measurements CoM axis Z.

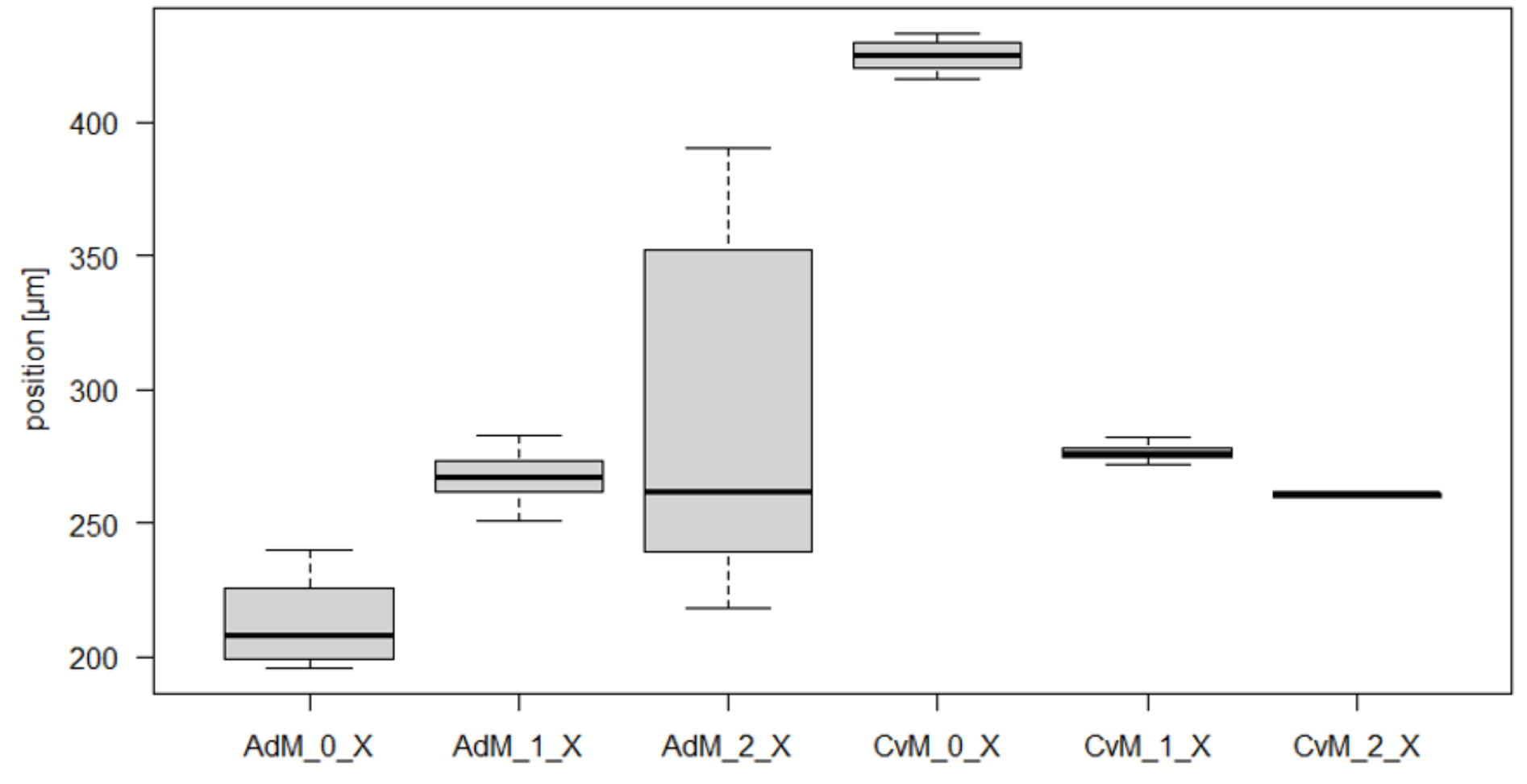

Figure 14

Measurements NcM axis X. 


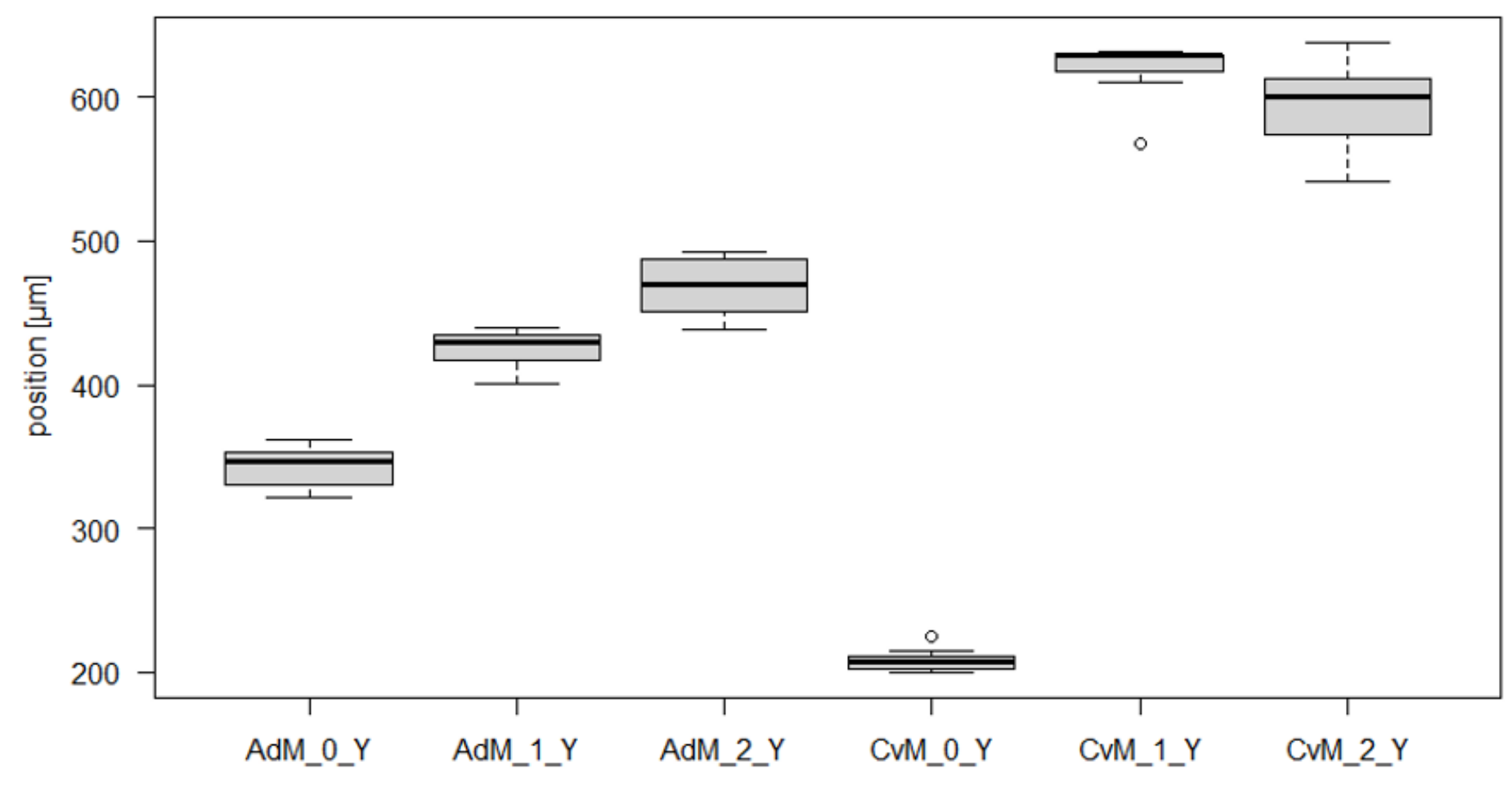

Figure 15

Measurements NcM axis Y.

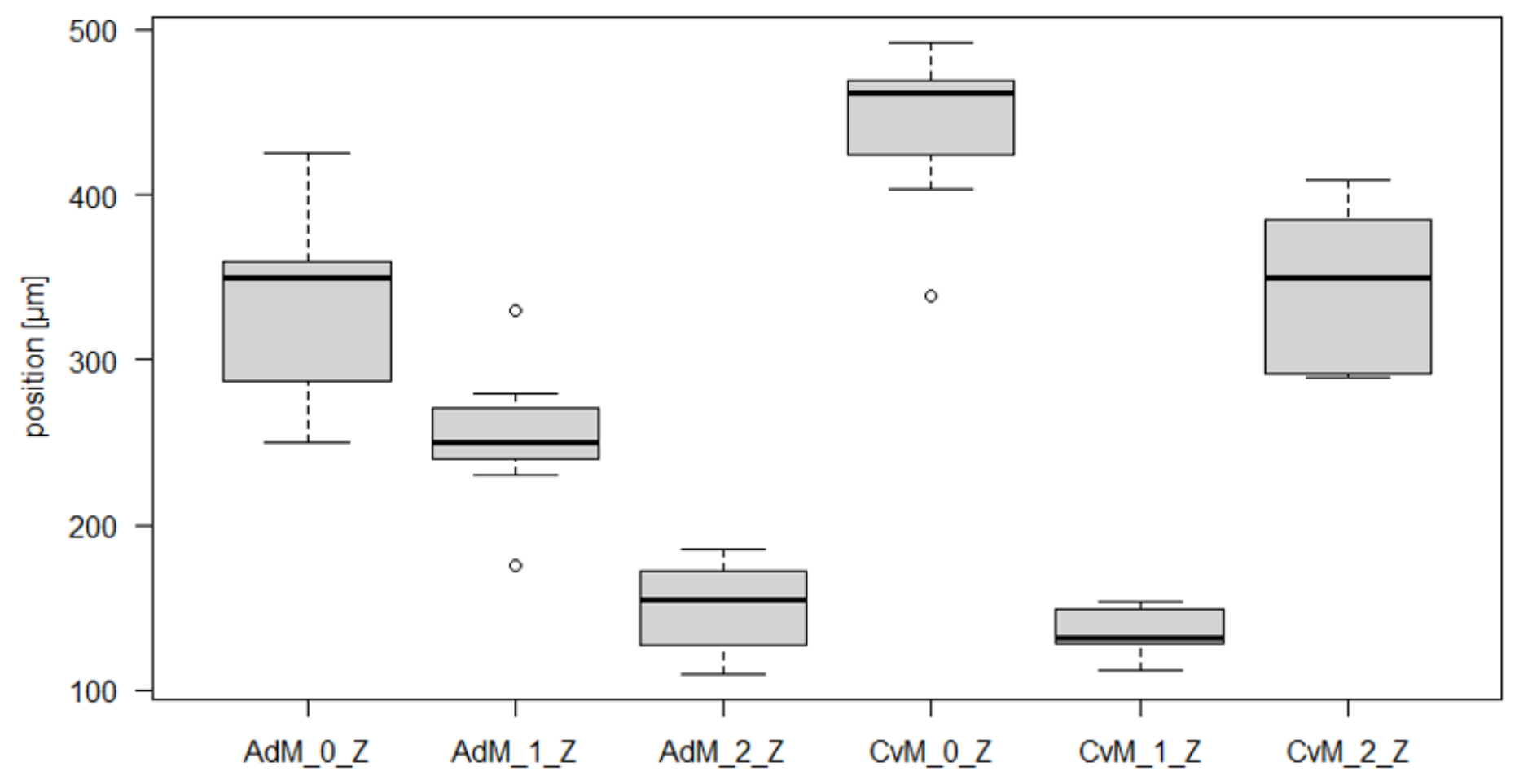

Figure 16

Measurements NcM axis Z. 